\title{
Mode interference in quantum joint probabilities for multimode Bose-condensed systems
}

\author{
V.I. Yukalov ${ }^{1,2}$, E.P. Yukalova ${ }^{1,3}$ and D. Sornette ${ }^{1,4}$ \\ ${ }^{1}$ D-MTEC, ETH Zürich, Swiss Federal Institute of Technology, \\ Zürich CH-8092, Switzerland \\ ${ }^{2}$ Bogolubov Laboratory of Theoretical Physics, \\ Joint Institute for Nuclear Research, Dubna 141980, Russia \\ ${ }^{3}$ Laboratory of Information Technologies, \\ Joint Institute for Nuclear Research, Dubna 141980, Russia \\ ${ }^{4}$ Swiss Finance Institute, c/o University of Geneva, \\ 40 blvd. Du Pont d'Arve, CH 1211 Geneva 4, Switzerland
}

\begin{abstract}
The method of defining quantum joint probabilities of two events is applied to a multimode system of trapped Bose-condensed atoms. The coherent modes are generated by modulating the trapping potential with an alternating field with a frequency in resonance with a transition frequency between two coherent modes. The system is subjected to nondestructive measurements randomly influencing the phase difference of the modes. The joint probability of observing two events, corresponding to the observation of two states, is calculated by employing the channel-state duality. The interference term in the joint probability can arise when the composite events form an entangled prospect and the system state is also entangled. This interference is due to the uncertainty induced by nondestructive measurements.
\end{abstract}

PACS: 03.65.Ta, 03.75.Gg, 67.85.Jk

Keywords: Bose-Einstein condensate, quantum joint probability, nondestructive measurements, coherent modes, interference effects

Corresponding author: V.I. Yukalov e-mail: yukalov@theor.jinr.ru 


\section{Introduction}

Multimode quantum systems provide efficient tools for quantum information processing and quantum computing [1,2]. Multimode systems are ubiquitous. These can be atomic systems with several populated electron energy levels, molecular ensembles with several rotovibrational modes, quantum dots with several exciton states, spin assemblies with several spin projections, trapped Bose-condensed atomic gases with several coherent modes, and so on [3]. The general requirement for creating such multimode systems is the existence of a discrete spectrum, which, in turn, usually requires the finiteness of the quantum system. The generation of several modes can be done by means of alternating resonance fields.

The probability of having a particular mode, at each moment of time, is characterized by fractional mode populations. But, for the purpose of quantum information processing, one may need to define the joint probability of observing two modes, or more generally, of two states that can be entangled. The aim of the present paper is to introduce, following the theory of quantum measurements for composite events [4], such a joint probability and to illustrate its properties by considering a two-mode system of trapped atoms at low temperature with weak interactions, when almost all atoms in an equilibrium trap would be in the Bose-Einstein condensate. Other modes can then be generated by a resonance trap modulation.

In Sec. 2, we define the joint probability of two modes by employing the channel-state duality based on the Choi-Jamiolkowski isomorphism. The general consideration is specified in Sec. 3 for a multi-mode Bose-condensed system of trapped atoms. In conclusion, we mention the analogies between the joint probabilities in the considered multimode system and the processes studied in quantum decision theory and the problem of creating quantum artificial intelligence.

\section{Multimode quantum systems}

A quantum system can be characterized by a set $\{|n\rangle\}$ of stationary solutions corresponding to the eigenvectors of the related stationary Hamiltonian. Such stationary solutions, associated with the eigenenergies $E_{n}$, are called modes, $n$ being a quantum multi-index labeling the stationary states. The closed linear envelope of these modes forms a Hilbert space

$$
\mathcal{H}=\operatorname{span}\{|n\rangle\} .
$$

The system state is its statistical operator that, for zero temperature, can be expressed as

$$
\hat{\rho}(t)=|\psi(t)\rangle\langle\psi(t)|
$$

through the wave functions

$$
|\psi(t)\rangle=\sum_{n} c_{n}(t)|n\rangle .
$$

The coefficients $c_{n}$ are normalized, such that their moduli squared give the fractional mode populations

$$
f_{n}(t) \equiv\left|c_{n}(t)\right|^{2}, \quad \sum_{n} f_{n}(t)=1 .
$$


We may define as a simple event $A_{n}$ the observation of the mode $|n\rangle$ at time $t$. According to the quantum theory of measurements [5], this event induces the correspondence

$$
A_{n} \rightarrow|n\rangle \rightarrow \hat{P}_{n} \equiv|n\rangle\langle n|
$$

where $\hat{P}_{n}$ is a projector. The probability of the event $A_{n}$ is

$$
p\left(A_{n}\right) \equiv \operatorname{Tr}_{\mathcal{H}} \hat{\rho}(t) \hat{P}_{n}=f_{n}(t),
$$

which, not surprisingly, coincides with the fractional mode population (4).

Let us now consider the case when more than a single measurement is done, namely a series of measurements are accomplished at times $0, t_{1}, t_{2}, \ldots, t_{0}, \ldots, t$. And suppose we wish to define the joint probability of two events, one being the event $A_{n}$ of observing the $n$-mode at time $t$, and another being an event $B_{\alpha}$ of observing a mode $|\alpha\rangle$ at a preceding time $t_{0}<t$. For the event $B_{\alpha}$, we have the correspondence

$$
B_{\alpha} \rightarrow|\alpha\rangle \rightarrow \hat{P}_{\alpha} \equiv|\alpha\rangle\langle\alpha|
$$

Generally, the modes at different times could be of different types, if the Hamiltonian has been changed. The events corresponding to the modes of different types can be termed incompatible. But even if the events, hence the modes, are compatible, that is, the modes are of the same type, it is convenient to distinguish the modes for time $t_{0}$ from the modes associated with time $t$.

The definition of quantum joint probability requires caution. It is easy to see that the Kirkwood [6] form does not constitute a probability, since for incompatible events it can be complex valued, while for compatible modes it reduces to the trivial expression

$$
\operatorname{Tr}_{\mathcal{H}} \hat{\rho}(t) \hat{P}_{n} \hat{P}_{\alpha}=\delta_{n \alpha}\left|c_{n}(t)\right|^{2} .
$$

Even more complicated is the problem of defining the quantum joint probability of two events, when one of them, say $B$, is not simple but consists of a union $\left\{B_{\alpha}\right\}$ of several events $B_{\alpha}$, so that the event $B$ corresponds to the observation of a multimode state

$$
|B\rangle=\sum_{\alpha} b_{\alpha}|\alpha\rangle, \quad B \equiv \biguplus_{\alpha} B_{\alpha}
$$

where $b_{\alpha}$ depends on time $t_{0}$. Then how could the joint probability of the events $A_{n}$ and $B$ be defined?

At this point, it is necessary to make some comments concerning notation. An event $B$ representing a set $\left\{B_{\alpha}\right\}$ could be denoted as $\bigcup_{\alpha} B_{\alpha}$, keeping in mind that this event induces the correspondence

$$
B \rightarrow|B\rangle \rightarrow \hat{P}_{B} \equiv|B\rangle\langle B| .
$$

But, if one were to forget about the above correspondence, then employing the notation $\bigcup_{\alpha} B_{\alpha}$ could lead to a confusion, if one would assume that $\bigcup_{\alpha} B_{\alpha}$ corresponded to $\sum_{\alpha} \hat{P}_{\alpha}$. In order to avoid such a confusion, we denote the complex event $B$ as is done in Eq. (8).

When one considers an explicitly prescribed dynamical picture, with the given evolution law and exactly formulated measurement procedures, then the whole system dynamics at times $0, t_{1}, t_{2}, \ldots, t_{0}, \ldots, t$ corresponds to the convolution of channels with the given states:

$$
\hat{\rho}_{0} \rightarrow \hat{\rho}_{1} \rightarrow \hat{\rho}_{2} \rightarrow \ldots \rightarrow \hat{\rho}_{t_{0}} \rightarrow \ldots \rightarrow \hat{\rho}_{t}
$$


Generally, the transformation of $\hat{\rho}_{k}$ to $\hat{\rho}_{k+1}$ is not unitary, since it may involve nonunitary actions of a measuring device. But the preceding states serve as initial conditions for the following states. Thus, if $\hat{\rho}_{t_{0}}$ is given, then it can be treated as an initial condition for $\hat{\rho}_{t}$, so that $\hat{\rho}_{t}=\hat{\rho}_{t}\left(\hat{\rho}_{t_{0}}\right)$. Then, the probability of two events $A_{n}$ and $B_{\alpha}$ in the dynamical channel picture is

$$
p_{n}(t)=\operatorname{Tr}_{\mathcal{H}} \hat{\rho}_{t}\left(\hat{\rho}_{t_{0}}\right) \hat{P}_{n}
$$

corresponding to the channel

$$
\left\{\mathcal{H}, \hat{\rho}_{t_{0}}\right\} \rightarrow\left\{\mathcal{H}, \hat{\rho}_{t}\left(\hat{\rho}_{t_{0}}\right)\right\}
$$

Separating in the probability (10) the term $f_{n}(t)$ due to the system unitary evolution, not perturbed by measurements, we have

$$
p_{n}(t)=f_{n}(t)+q_{n}(t)
$$

The above channel picture, according to the Choi-Jamiolkowski isomorphism [7,8] can be equivalently represented as a composite event in a composite system constructed as follows. Let the event $A_{n}$ be the observation of an $n$-mode at time $t$ and the event $B_{\alpha}$ be the observation of an $\alpha$-mode at a preceding time $t_{0}<t$. We can define the Hilbert spaces

$$
\mathcal{H}_{A} \equiv \operatorname{span}\{|n\rangle\}, \quad \mathcal{H}_{B} \equiv \operatorname{span}\{|\alpha\rangle\}
$$

and introduce their tensor product

$$
\mathcal{H}_{A B} \equiv \mathcal{H}_{A} \bigotimes \mathcal{H}_{B}=\operatorname{span}\{|n \alpha\rangle \equiv|n\rangle \otimes|\alpha\rangle\}
$$

The composite event of observing $A_{n}$ and $B_{\alpha}$ is denoted as $A_{n} \otimes B_{\alpha}$ that induces the correspondence

$$
A_{n} \bigotimes B_{\alpha} \rightarrow \hat{P}_{n} \bigotimes \hat{P}_{\alpha}=|n \alpha\rangle\langle n \alpha|
$$

The joint probability of these events is

$$
p\left(A_{n} \bigotimes B_{\alpha}\right) \equiv \operatorname{Tr}_{A B} \hat{\rho}_{A B} \hat{P}_{n} \bigotimes \hat{P}_{\alpha}
$$

where the trace is over the space (14).

If the matrix elements of $\hat{\rho}_{A B}$ have the form

$$
\rho_{m n}^{\alpha \beta} \equiv\left\langle m \alpha\left|\hat{\rho}_{A B}\right| n \beta\right\rangle=c_{m \alpha} c_{n \beta}^{*},
$$

then the probability (16) becomes

$$
p\left(A_{n} \bigotimes B_{\alpha}\right)=\left\langle n \alpha\left|\hat{\rho}_{A B}\right| n \alpha\right\rangle=\left|c_{n \alpha}\right|^{2} .
$$

For the correct normalization of the probability, such that

$$
\begin{gathered}
p\left(A_{n}\right)=\sum_{\alpha} p\left(A_{n} \bigotimes B_{\alpha}\right), \quad p\left(B_{\alpha}\right)=\sum_{n} p\left(A_{n} \bigotimes B_{\alpha}\right), \\
\sum_{n} p\left(A_{n}\right)=\sum_{\alpha} p\left(B_{\alpha}\right)=1,
\end{gathered}
$$


the properties

$$
\begin{gathered}
\sum_{\alpha}\left|c_{n \alpha}\right|^{2}=\left|c_{n}\right|^{2}, \quad \sum_{n}\left|c_{n \alpha}\right|^{2}=\left|c_{\alpha}\right|^{2}, \\
\sum_{n \alpha}\left|c_{n \alpha}\right|^{2}=\sum_{n}\left|c_{n}\right|^{2}=\sum_{\alpha}\left|c_{\alpha}\right|^{2}=1,
\end{gathered}
$$

are required.

The Choi-Jamiolkowski isomorphism establishes the channel-state duality, according to which channel (11) is isomorphic to the composite system

$$
\left\{\mathcal{H}_{A B}, \hat{\rho}_{A B}\right\}
$$

with the composite state $\hat{\rho}_{A B}$. The joint probability of two simple events is given by Eq. (18). It is then straightforward to introduce the conditional quantum probability

$$
p\left(A_{n} \mid B_{\alpha}\right) \equiv \frac{p\left(A_{n} \otimes B_{\alpha}\right)}{p\left(B_{\alpha}\right)}
$$

resorting to the Bayesian rule.

The composite event $A_{n} \otimes B_{\alpha}$, being the tensor product of two simple events, is called factorized. A more complicated structure arises when one of the events is not simple but is a union of several events. Let us consider the event $B=\biguplus_{\alpha} B_{\alpha}$ of having the multimode state $|B\rangle$ at time $t_{0}$. Then the composite event

$$
\pi_{n}=A_{n} \bigotimes \biguplus_{\alpha} B_{\alpha}
$$

represents an entangled prospect inducing the correspondence

$$
\pi_{n} \rightarrow\left|\pi_{n}\right\rangle \equiv|n\rangle \bigotimes|B\rangle \rightarrow \hat{P}\left(\pi_{n}\right)
$$

where

$$
\hat{P}\left(\pi_{n}\right) \equiv\left|\pi_{n}\right\rangle\left\langle\pi_{n}\right|
$$

is a prospect operator. The latter is required to satisfy the resolution of unity

$$
\sum_{n} \hat{P}\left(\pi_{n}\right)=\hat{1}_{A B}
$$

with $\hat{1}_{A B}$ being the identity operator on the space (14). By definition, a prospect operator $\hat{P}\left(\pi_{n}\right)$ is positive, but it is not necessarily a projector. The family $\left\{\hat{P}\left(\pi_{n}\right)\right\}$ of positive operators, satisfying resolution (24), forms a positive operator-valued measure [9, 10].

The prospect probability is

$$
p\left(\pi_{n}\right) \equiv \operatorname{Tr}_{A B} \hat{\rho}_{A B} \hat{P}\left(\pi_{n}\right)
$$

Separating here the diagonal and nondiagonal parts with respect to the indices $\alpha$ and $\beta$, we obtain

$$
p\left(\pi_{n}\right)=f\left(\pi_{n}\right)+q\left(\pi_{n}\right)
$$


where

$$
f\left(\pi_{n}\right)=\sum_{\alpha}\left|b_{\alpha}\right|^{2} p\left(A_{n} \bigotimes B_{\alpha}\right), \quad q\left(\pi_{n}\right)=\sum_{\alpha \neq \beta} b_{\alpha}^{*} b_{\beta} c_{n \alpha} c_{n \beta}^{*}
$$

In view of resolution (24), the probability (25) is normalized:

$$
\sum_{n} p\left(\pi_{n}\right)=1
$$

The term $f\left(\pi_{n}\right)$ describes the quasiclassical prospect probability that is also normalized as

$$
\sum_{n} f\left(\pi_{n}\right)=\sum_{\alpha}\left|b_{\alpha}\right|^{2} p\left(B_{\alpha}\right)=1
$$

And the second term in the right-hand side of the probability (26) is the interference term caused by the mode interference. Because of normalizations (28) and (29), we have

$$
\sum_{n} q\left(\pi_{n}\right)=0
$$

According to the channel-state duality, the prospect probabilities in the channel picture and in the composite-state representation must coincide:

$$
p\left(\pi_{n}\right)=p_{n}(t)
$$

Let us consider the case where the modes of the multimode state (8) are equally weighted, that is, $\left|b_{\alpha}\right|^{2}=$ const, which, in view of normalizations (19) and (29) gives $\left|b_{\alpha}\right|^{2}=1$. Then from Eq. (27) it follows that

$$
f\left(\pi_{n}\right)=\left|c_{n}\right|^{2}=f_{n}(t),
$$

as defined by expression (4). Therefore, for the interference term, we have

$$
q\left(\pi_{n}\right)=q_{n}(t)
$$

which allows us to calculate this term by employing the dynamical channel picture.

We may notice that the interference term disappears when the system state is not entangled, having the form

$$
\hat{\rho}_{A B}=\sum_{n \alpha}\left|c_{n \alpha}\right|^{2}|n \alpha\rangle\langle n \alpha|
$$

Hence, state entanglement is a necessary condition for a nonzero interference term. But this is not a sufficient condition. For instance, the maximally entangled Bell state

$$
\hat{\rho}_{A B}=\frac{1}{M} \sum_{m n}|m m\rangle\langle n n|
$$

yields $q\left(\pi_{n}\right)=0$.

The Bell state is entangled and is also generating entanglement. The measure of entanglement production is defined [11] as

$$
\varepsilon\left(\hat{\rho}_{A B}\right) \equiv \log \frac{\left\|\hat{\rho}_{A B}\right\|}{\left\|\hat{\rho}_{A}\right\|\left\|\hat{\rho}_{B}\right\|},
$$


where

$$
\hat{\rho}_{A} \equiv \operatorname{Tr}_{B} \hat{\rho}_{A B}, \quad \hat{\rho}_{B} \equiv \operatorname{Tr}_{A} \hat{\rho}_{A B}
$$

For the Bell state with $M$ modes, this gives $\varepsilon\left(\hat{\rho}_{A B}\right)=\log M$. However, the interference term is zero. Summarizing the above properties, we come to the following conclusion.

Proposition. For the interference term $q\left(\pi_{n}\right)$ to be nonzero, it is necessary (but not sufficient) that the prospect $\pi_{n}$ be entangled and the state $\hat{\rho}_{A B}$ be entangled.

\section{Trapped Bose-condensed gas}

As a concrete illustration of the above theory, we consider the system of trapped Bose atoms that weakly interact with each other, so that

$$
\rho^{1 / 3} a_{s} \ll 1
$$

where $\rho$ is the mean atomic density and $a_{s}$ is the scattering length. At low temperature $T \rightarrow 0$, almost all atoms pile down to the ground state, thus forming a Bose-Einstein condensate corresponding to an atomic coherent state. The properties of such atomic gases have been intensively discussed in several books [12 15] and review articles [16 30].

The system is characterized by the coherent field $\eta(\mathbf{r}, t)$ normalized to the number of atoms

$$
N=\int|\eta(\mathbf{r}, t)|^{2} d \mathbf{r}
$$

The coherent field plays the role of the condensate wave function satisfying the nonlinear Schrödinger equation

$$
i \frac{\partial}{\partial t} \eta(\mathbf{r}, t)=\hat{H}(\mathbf{r}, t) \eta(\mathbf{r}, t)
$$

with the nonlinear Hamiltonian

$$
\hat{H}(\mathbf{r}, t)=-\frac{\nabla^{2}}{2 m}+U(\mathbf{r}, t)+\Phi_{0}|\eta(\mathbf{r}, t)|^{2},
$$

in which $\Phi_{0} \equiv 4 \pi a_{s} / m$ is the effective interaction strength. Here and in what follows, the Planck constant is set to one.

The external potential consists of two parts,

$$
U(\mathbf{r}, t)=U(\mathbf{r})+V(\mathbf{r}, t)
$$

of a stationary trapping potential $U(\mathbf{r})$ and of an additional time-dependent potential of trap modulation, which can be taken in the form

$$
V(\mathbf{r}, t)=V_{1}(\mathbf{r}) \cos (\omega t)+V_{2}(\mathbf{r}) \sin (\omega t) .
$$

It is convenient to make the replacement

$$
\eta(\mathbf{r}, t)=\sqrt{N} \varphi(\mathbf{r}, t)
$$

introducing the function that is normalized to one:

$$
\int|\varphi(\mathbf{r}, t)|^{2} d \mathbf{r}=1 .
$$


Then, Eq. (38) transforms into

$$
i \frac{\partial}{\partial t} \varphi(\mathbf{r}, t)=\left[\hat{H}_{0}(\mathbf{r})+V(\mathbf{r}, t)\right] \varphi(\mathbf{r}, t)
$$

where the stationary nonlinear Hamiltonian is

$$
\hat{H}_{0}(\mathbf{r})=-\frac{\nabla^{2}}{2 m}+U(\mathbf{r})+N \Phi_{0}|\varphi(\mathbf{r}, t)|^{2} .
$$

The coherent modes are defined [31] as the solutions to the stationary eigenvalue problem

$$
\hat{H}_{0}(\mathbf{r}) \varphi_{n}(\mathbf{r})=E_{n} \varphi_{n}(\mathbf{r})
$$

The solution of the time-dependent equation (44) can be represented as an expansion over the coherent modes,

$$
\varphi(\mathbf{r}, t)=\sum_{n} c_{n}(t) \varphi_{n}(\mathbf{r}) e^{-i E_{n} t}
$$

Substituting this expansion into Eq. (44) yields the equations for the coefficient functions $c_{n}(t)$. We assume that, at the initial time, practically the whole system is in a coherent condensed state and only one more level with the energy $E_{2}$ could be slightly populated, so that the initial condition is

$$
c_{n}(0)=c_{1}(0) \delta_{n 1}+c_{2}(0) \delta_{n 2}
$$

The excited level with $E_{2}$ is selected by the use of the resonance alternating field with the frequency

$$
\omega=E_{2}-E_{1}
$$

that is in resonance with the corresponding transition frequency.

Under the initial condition (48) and the resonance condition (49), only two modes are involved in the dynamics resulting in the equations

$$
i \frac{d c_{1}}{d t}=\alpha_{12}\left|c_{2}\right|^{2} c_{1}+\frac{1}{2} \beta_{12} c_{2}, \quad i \frac{d c_{2}}{d t}=\alpha_{21}\left|c_{1}\right|^{2} c_{2}+\frac{1}{2} \beta_{12}^{*} c_{1}
$$

in which the notations

$$
\begin{gathered}
\alpha_{m n} \equiv N \Phi_{0} \int\left|\varphi_{m}(\mathbf{r})\right|^{2}\left[2\left|\varphi_{n}(\mathbf{r})\right|^{2}-\left|\varphi_{m}(\mathbf{r})\right|^{2}\right] d \mathbf{r} \\
\beta_{m n} \equiv \int \varphi_{m}^{*}(\mathbf{r})\left[V_{1}(\mathbf{r})-i V_{2}(\mathbf{r})\right] \varphi_{n}(\mathbf{r}) d \mathbf{r}
\end{gathered}
$$

are used. In what follows, we shall need the parameters

$$
\alpha \equiv \frac{1}{2}\left(\alpha_{12}+\alpha_{21}\right), \quad \beta \equiv\left|\beta_{12}\right|, \quad \delta \equiv \frac{1}{2}\left(\alpha_{12}-\alpha_{21}\right) .
$$

Let us make the transformation

$$
c_{1}=\sqrt{\frac{1-s}{2}} e^{i \varepsilon_{1} t}, \quad c_{2}=\sqrt{\frac{1+s}{2}} e^{i \varepsilon_{2} t}
$$


defining the population imbalance $s$ and the phase difference $x$ by the corresponding expressions

$$
s \equiv\left|c_{2}\right|^{2}-\left|c_{1}\right|^{2}, \quad x \equiv \varepsilon_{1}-\varepsilon_{2}
$$

Then, Eqs. (50) reduce to

$$
\frac{d s}{d t}=-\beta \sqrt{1-s^{2}} \sin x, \quad \frac{d x}{d t}=\alpha s+\frac{\beta s}{\sqrt{1-s^{2}}} \cos x+\delta .
$$

In what follows, we keep in mind repulsive interactions, because of which the parameter $\alpha$ is positive. The parameter $\delta$ is small and can be omitted.

It is convenient to measure time in units of $1 / \alpha$ and to introduce the pumping parameter $b \equiv \beta / \alpha$ characterizing the strength of the modulation field as compared to that of the effective atomic interactions. We assume that the observation of coherent modes is accomplished by means of nondestructive measurements that influence only the phases of the modes, so that a measurement leads to a random shift of the phase of each mode. The evolution equations (54) become

$$
\frac{d s}{d t}=-b \sqrt{1-s^{2}} \sin x, \quad d x=s\left(1+\frac{b}{\sqrt{1-s^{2}}} \cos x\right) d t+\sigma d W_{t},
$$

where $W_{t}$ is the standard Wiener process and $\sigma$ is the standard deviation related to the random noise.

Equations (55) without noise exhibit solutions of two different types, depending on the value of the pumping parameter $b$. The dynamic transition between the qualitatively different regimes of motion happens on the critical surface given by the separatrix

$$
2 b_{c}\left(1+\sqrt{1-s_{0}^{2}} \cos x_{0}\right)=s_{0}^{2},
$$

where the critical $b_{c}$ depends on the initial conditions $s_{0}=s(0), x_{0}=x(0)$. In the subcritical regime, or mode locked regime, when $b<b_{c}$, the modes experience weak Rabi oscillations close to their initial values and the population imbalance never crosses the zero line, so that, if $s(0)<0$ then

$$
-1 \leq s(t)<0 \quad\left(b<b_{c}\right) .
$$

In the supercritical regime, or mode unlocked regime, when $b>b_{c}$, the modes display Josephson oscillations, such that the population imbalance varies in the whole range of its validity:

$$
-1 \leq s(t) \leq 1 \quad\left(b>b_{c}\right)
$$

In the presence of random perturbations, transitions between these regimes can happen, which we study numerically.

We solve Eqs. (55) for $s(t)$ and $x(t)$, which define the mode probabilities

$$
p_{1}(t)=\frac{1-s(t)}{2}, \quad p_{2}(t)=\frac{1+s(t)}{2} .
$$

The unperturbed fractional mode populations are given by

$$
f_{n}(t)=\lim _{\sigma \rightarrow 0} p_{n}(t) .
$$


The interference terms are defined by the expression

$$
q_{n}(t)=p_{n}(t)-f_{n}(t)
$$

We also study the behavior of the average interference terms

$$
\bar{q}_{n}(t) \equiv \frac{1}{t} \int_{0}^{t} q_{n}(\tau) d \tau
$$

Because of the relations

$$
p_{1}(t)+p_{2}(t)=1, \quad q_{1}(t)+q_{2}(t)=0,
$$

it is sufficient to show the behavior of only $p_{1}(t), q_{1}(t)$, and $\bar{q}_{1}(t)$. The corresponding temporal evolution of these quantities are presented in Figs. 1 and 2 for subcritical regime and in Figs. 3 and 4 for supercritical regime. In all the cases, we take the initial conditions $s_{0}=-0.9$ and $x_{0}=0$, corresponding to the situation when, at $t=0$, almost all atoms are Bose-condensed. The probabilities, as well as interference terms, strongly fluctuate. The average interference terms at the beginning fluctuate, tending to a constant at large time $t \rightarrow \infty$.

In order to find out the limit of the average interference terms, let us consider the case of a small pumping parameter $b \ll 1$. Then, as follows from Eqs. (55), the variable $s$ can be treated as slow and $x$ as fast. In such a case, we can resort to the averaging techniques [32,33].

Generally, by definition (59), we have

$$
q_{1}(t)=\frac{1}{2}\left[s^{(0)}(t)-s(t)\right]
$$

where

$$
s^{(0)}(t) \equiv \lim _{\sigma \rightarrow 0} s(t) .
$$

When, there is no pumping, so that $b=0$, then $s(t)=s_{0}$. To find the behavior of $s(t)$ in the first order with respect to $b$, we need to get $x$ in the zero order with respect to $b$. In the zero order in $b$, the second of equations (55) simplifies into

$$
d x=s_{0} d t+\sigma d W_{t}
$$

Under the initial condition $x(0)=0$, its solution reads

$$
x=s_{0} t+\sigma W_{t} .
$$

Substituting this into the equation for the slow variable and averaging the right-hand side over stochastic fluctuations, we have

$$
\frac{d s}{d t}=-b \sqrt{1-s_{0}^{2}}\langle\langle\sin x\rangle\rangle
$$

with the double angle brackets representing the averaging over these fluctuations.

Using the property

$$
\left\langle\left\langle\exp \left(i \sigma W_{t}\right)\right\rangle\right\rangle=\exp \left\{-\frac{\sigma^{2}}{2}\left\langle\left\langle W_{t}^{2}\right\rangle\right\rangle\right\}
$$


with $\left\langle\left\langle W_{t}^{2}\right\rangle\right\rangle=t$, we obtain

$$
\left\langle\left\langle\sin \left(s_{0} t+\sigma W_{t}\right)\right\rangle\right\rangle=\sin \left(s_{0} t\right) e^{-\sigma^{2} t / 2} .
$$

Consequently, Eq. (65) transforms into

$$
\frac{d s}{d t}=-b \sqrt{1-s_{0}^{2}} \sin \left(s_{0} t\right) e^{-\sigma^{2} t / 2} .
$$

The solution to this equation, with the initial condition $s(0)=s_{0}$, reads as

$$
s=s_{0}+\frac{4 b \sqrt{1-s_{0}^{2}}}{4 s_{0}^{2}+\sigma^{4}}\left\{\left[s_{0} \cos \left(s_{0} t\right)+\frac{\sigma^{2}}{2} \sin \left(s_{0} t\right)\right] e^{-\sigma^{2} t / 2}-s_{0}\right\} .
$$

Respectively, solution (62) becomes

$$
s^{(0)}=s_{0}+\frac{b \sqrt{1-s_{0}^{2}}}{s_{0}}\left[\cos \left(s_{0} t\right)-1\right] .
$$

Averaging over time, we find

$$
\begin{aligned}
& \lim _{t \rightarrow \infty} \frac{1}{t} \int_{0}^{t} s(\tau) d \tau=s_{0}\left(1-\frac{4 b \sqrt{1-s_{0}^{2}}}{4 s_{0}^{2}+\sigma^{4}}\right), \\
& \lim _{t \rightarrow \infty} \frac{1}{t} \int_{0}^{t} s^{(0)}(\tau) d \tau=s_{0}\left(1-\frac{b \sqrt{1-s_{0}^{2}}}{s_{0}^{2}}\right) .
\end{aligned}
$$

Hence, the limit of the time-averaged quantity (61) is

$$
\lim _{t \rightarrow \infty} \bar{q}_{1}(t)=-\frac{b \sigma^{4} \sqrt{1-s_{0}^{2}}}{2 s_{0}\left(4 s_{0}^{2}+\sigma^{4}\right)} .
$$

Because of condition (30), we have $\bar{q}_{2}(\infty)=-\bar{q}_{1}(\infty)$.

When $b / \sigma \rightarrow 0$, the limit $\bar{q}_{1}(\infty)=0$, since, as has been mentioned above, there is no dynamics in the system. In the opposite case, when $\sigma / b \rightarrow 0$, again $\bar{q}_{1}(\infty)=0$, because the measurements do not induce mode interference. In the intermediate situation, when neither $b$ nor $\sigma$ are zero, the interference term also is not zero. For example, for $b=0.25, s_{0}=-0.9$ and $\sigma=0.5$, expression (70) yields $\lim _{t \rightarrow \infty} \bar{q}_{1}(t)=0.001146$. In the limit of large-noise amplitude $\sigma \rightarrow+\infty$, we have $\lim _{t \rightarrow \infty} \bar{q}_{1}(t)=-\frac{b \sqrt{1-s_{0}^{2}}}{2 s_{0}}$. For $b=0.25$ and $s_{0}=-0.9$, this gives $\bar{q}_{1}(\infty)=0.06054$.

\section{Conclusion}

We have presented a general formulation of the quantum joint probability for a multimode system, based on the Choi-Jamiolkowski isomorphism between the channel picture and a composite system, via tensorial products of Hilbert spaces. We have shown that, in order for the interference terms to be present, it is essential that these states be entangled, 
which is achievable by means of modern techniques [34,35]. As has been mentioned in the Introduction, multimode states can be created in a variety of experimental setups.

We have applied this formalism to a two-mode system of trapped Bose-condensed atoms under the influence of an oscillatory resonant trap modulation field. Assuming non-destructive measurements, whose impact is only to scramble partially the phases, we have obtained the dynamics of the population imbalance $s(t)$ between the two modes and of the interference terms $q_{1}(t)=-q_{2}(t)$. In particular, we find a non-zero long-term average of the interference terms, as long as the pumping parameter $b$ and the standard deviation $\sigma$ of the random noise are non zero.

The population imbalance $s(t)$ allows us to find the probability $p_{1}(t)=(1-s(t)) / 2$ of observing the first mode at time $t$, while being subject, at previous times, to measuring perturbations modeled by random noise in the evolution of the phase difference. Because of the random noise, the state at time $t_{0}<t$ is not exactly known, and is assumed to be a twomode state with equal weights. In that sense, finding $p_{1}(t)$ corresponds to a measurement under uncertainty. On the other side, the certain measurement is associated with defining the fractional mode population $f_{1}(t)$ calculated for the case without noise. The difference $q_{1}(t)=p_{1}(t)-f_{1}(t)$ in this dynamical picture imitates the behavior of the interference term $q\left(\pi_{1}\right)$, which is justified by the channel-state duality.

We would like to mention that measurements under uncertainty are analogous to taking decisions under uncertainty, as studied in decision making. In quantum decision theory [36 39], taking decisions under uncertainty is also accompanied by the appearance of interference terms corresponding to deliberations in the evaluation of several admissible choices. The correspondence suggests that the uncertainty existing in decision making may be interpreted as hidden nondestructive measurements and processes occurring in the brain, which is continuously exposed to exogenous influences. The existence of such interference has also to be taken into account in quantum information processing and the problem of creating quantum artificial intelligence [40]. In quantum decision theory, the terms $q\left(\pi_{n}\right)$ are interpreted as attraction factors, describing the attitude of a decision maker to the available prospects. A negative attraction factor implies subconscious repulsion of the decision maker to the considered prospect, while a positive attraction factor is associated with the decision maker subconscious attraction to the prospect.

In that sense, treating the considered model as a cartoon of a functioning intelligence, it is possible to give the following interpretation for the signs of term (70). First, remembering that $s_{0}<0$, we see that $\bar{q}_{1}(\infty)$ is positive, which increases the probability of the system to be in the ground state. In other words, in the presence of the mode interference caused by measurements, the ground state is more attractive for the system. On the contrary, the negative value $\bar{q}_{2}(\infty)$, related to the excited upper mode, demonstrates a negative attitude of the system to the population of the higher mode in the presence of random perturbations.

In the framework of quantum decision theory [36 39], it was shown that a non-informative prior leads to the quarter law, when the average absolute value of the interference factor $q$ is equal to $1 / 4$. Considering the present two-mode system of trapped Bose-condensed atoms under the influence of an oscillating resonant trap modulation as a cartoon model of functioning decision making, this would lead, in the limit of large noise amplitude $\sigma \rightarrow+\infty$, to the relationship $s_{0}^{2}=4 b^{2} /\left(4 b^{2}+1\right)$, for which $\lim _{t \rightarrow \infty} \bar{q}_{1}(t)=1 / 4$. In other words, this suggests the existence of a non trivial relationship between the initial state of the deciding mind and the coupling with the external world. While being very tentative, this opens the 
road towards a dynamical approach to quantum decision theory. Going beyond the noninformative prior, a priori knowledge could be encoded into different initial states, leading to different values of the attraction factors. These analogies suggest that cold trapped bosons in the Bose-condensed state could be used for quantum information processing and for creating quantum artificial intelligence.

\section{Acknowledgments}

Financial support from the Swiss National Foundation and from the Russian Foundation for Basic Research are appreciated. 


\section{References}

[1] Nielsen M and Chuang I 2000 Quantum Computation and Quantum Information (Cambridge: Cambridge University)

[2] Keyl M 2002 Phys. Rep. 369431

[3] Birman J L, Nazmitdinov R G and Yukalov V I 2013 Phys. Rep. 5261

[4] Yukalov V I and Sornette D 2013 Laser Phys. 23105502

[5] von Neumann J 1955 Mathematical Foundations of Quantum Mechanics (Princeton: Princeton University)

[6] Kirkwood J G 1933 Phys. Rev. 4431

[7] Choi M D 1972 Can. J. Math. 24520

[8] Jamiolkowski A 1972 Rep. Math. Phys. 3275

[9] Holevo A S 2011 Probabilistic and Statistical Aspects of Quantum Theory (Berlin: Springer)

[10] Holevo A S and Giovannetti V 2012 Rep. Prog. Phys. 75046001

[11] Yukalov V I 2003 Phys. Rev. A 68022109

[12] Lieb E H, Seiringer R, Solovej J P and Yngvason J 2005 The Mathematics of the Bose Gas and its Condensation (Basel: Birkháuser)

[13] Pitaevskii L and Stringari S 2003 Bose-Einstein Condensation (Oxford: Clarendon)

[14] Letokhov V 2007 Laser Control of Atoms and Molecules (New York: Oxford University Press)

[15] Pethick C J and Smith H 2008 Bose-Einstein Condensation in Dilute Gases (Cambridge: Cambridge University Press)

[16] Courteille P W, Bagnato V S and Yukalov V I 2001 Laser Phys. 11659

[17] Andersen J O 2004 Rev. Mod. Phys. 76599

[18] Yukalov V I 2004 Laser Phys. Lett. 1435

[19] Bongs K and Sengstock K 2004 Rep. Prog. Phys. 67907

[20] Yukalov V I and Girardeau M D 2005 Laser Phys. Lett. 2375

[21] Morsch O and Oberthaler M 2006 Rev. Mod. Phys. 78179

[22] Posazhennikova A 2006 Rev. Mod. Phys. 781111

[23] Yukalov V I 2007 Laser Phys. Lett. 4632 
[24] Proukakis N P and Jackson B 2008 J. Phys. B: At. Mol. Opt. Phys. 41203002

[25] Yurovsky V A, Olshanii M and Weiss D S 2008 Adv. At. Mol. Opt. Phys. 5561

[26] Moseley C, Fialko O and Ziegler K 2008 Ann. Phys. (Berlin) 17561

[27] Bloch I, Dalibard J and Zwerger W 2008 Rev. Mod. Phys. 80885

[28] Yukalov V I 2009 Laser Phys. 191

[29] Yukalov V I 2011 Phys. Part. Nucl. 42460

[30] Yukalov V I 2012 Laser Phys. 221145

[31] Yukalov V I, Yukalova E P and Bagnato V S 1997 Phys. Rev. A 564845

[32] Bogolubov N N and Mitropolsky Y A 1961 Asymptotic Methods in the Theory of Nonlinear Oscillations (New York: Gordon and Breach)

[33] Yukalov V I and Yukalova E P 2000 Phys. Part. Nucl. 31561

[34] Dobek K, Karpinski M, Demkowicz-Dobrzanski R, Banaszek K and Horodecki P 2013 Laser Phys. 23025204

[35] Zha X, Yuan C and Zhang Y 2013 Laser Phys. Lett. 10045201

[36] Yukalov V I and Sornette D 2008 Phys. Lett. A 3726867

[37] Yukalov V I and Sornette D 2009 Eur. Phys. J. B 71533

[38] Yukalov V I and Sornette D 2009 Entropy 111073

[39] Yukalov V I and Sornette D 2010 Adv. Complex Syst. 13659

[40] Yukalov V I and Sornette D 2009 Laser Phys. Lett. 6833 


\section{Figure Captions}

Fig.1 Subcritical regime with $b=0.25<b_{c}=0.282$. Population imbalance $s(t)$ and phase difference $x(t)$, as functions of dimensionless time, for $\sigma=0.5$ (solid line) and $\sigma=0$ (dashed line).

Fig.2 Subcritical regime with $b=0.25<b_{c}=0.282$. Ground-state probability $p_{1}(t)$ for $\sigma=0.5$ (solid line) and $\sigma=0$ (dashed line); interference factor $q_{1}(t)$ and mean interference factor $\bar{q}_{1}(t)$ for $\sigma=0.5$.

Fig.3 Supercritical regime with $b=0.5>b_{c}=0.282$. Population imbalance $s(t)$ and phase difference $x(t)$, as functions of dimensionless time, for $\sigma=0.5$ (solid line) and $\sigma=0$ (dashed line).

Fig.4 Supercritical regime with $b=0.5>b_{c}=0.282$. Ground-state probability $p_{1}(t)$ for $\sigma=0.5$ (solid line) and $\sigma=0$ (dashed line); interference factor $q_{1}(t)$ and mean interference factor $\bar{q}_{1}(t)$ for $\sigma=0.5$. 

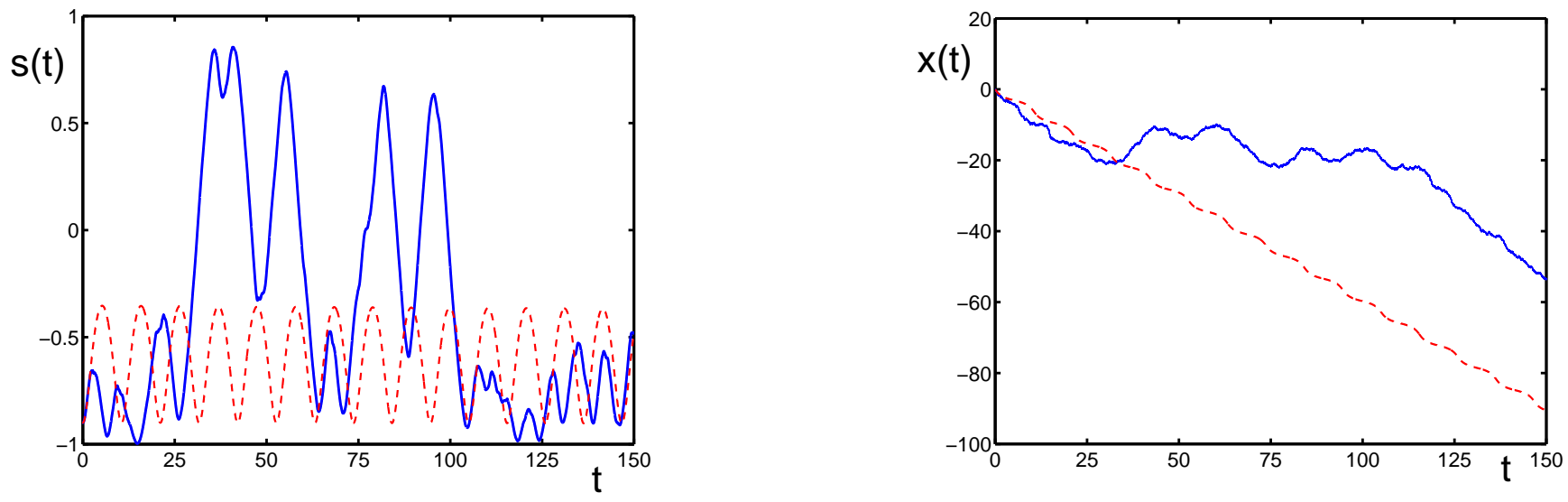

Figure 1: Subcritical regime with $b=0.25<b_{c}=0.282$. Population imbalance $s(t)$ and phase difference $x(t)$, as functions of dimensionless time, for $\sigma=0.5$ (solid line) and $\sigma=0$ (dashed line). 

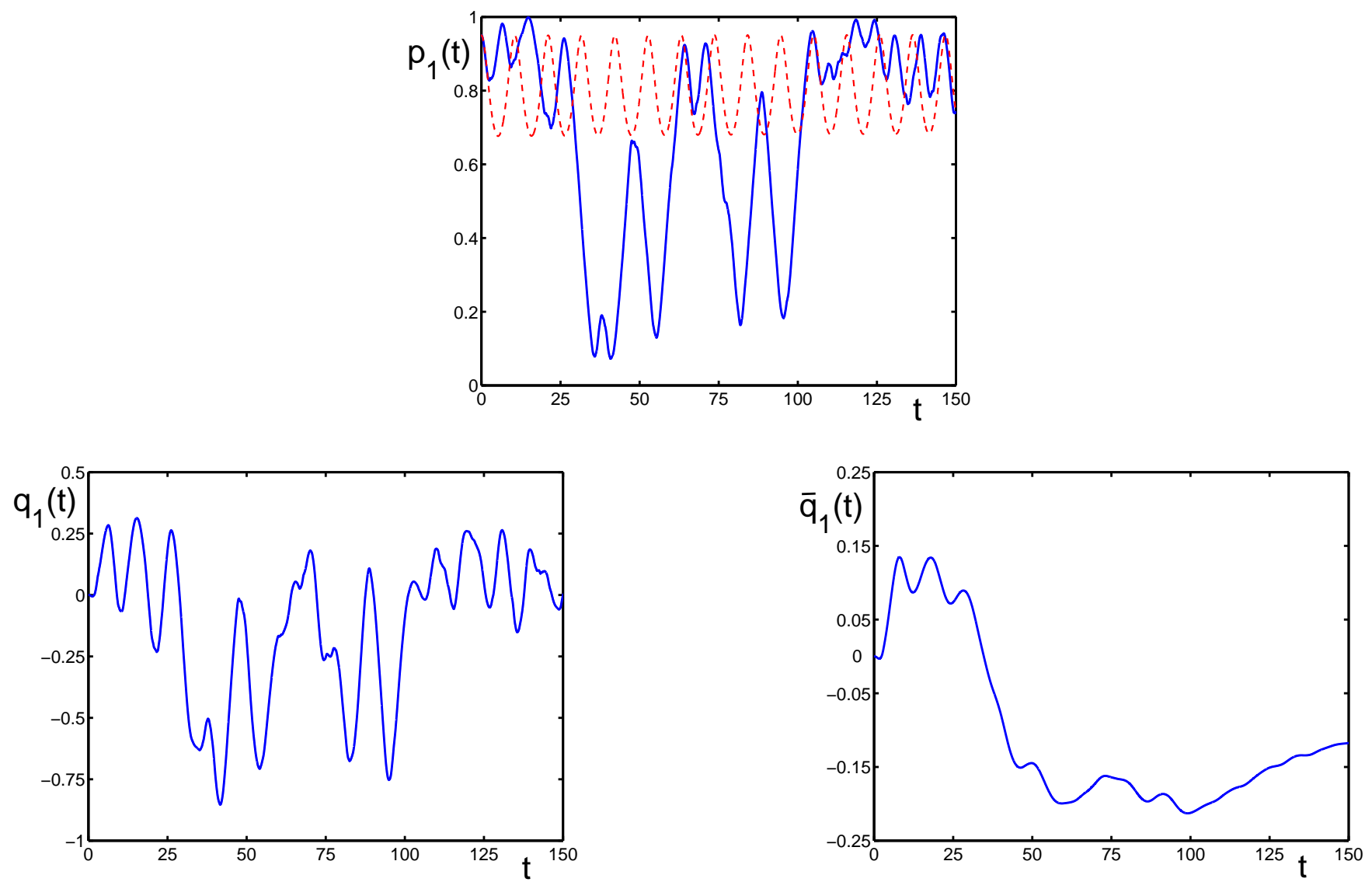

Figure 2: Subcritical regime with $b=0.25<b_{c}=0.282$. Ground-state probability $p_{1}(t)$ for $\sigma=0.5$ (solid line) and $\sigma=0$ (dashed line); interference factor $q_{1}(t)$ and mean interference factor $\bar{q}_{1}(t)$ for $\sigma=0.5$. 

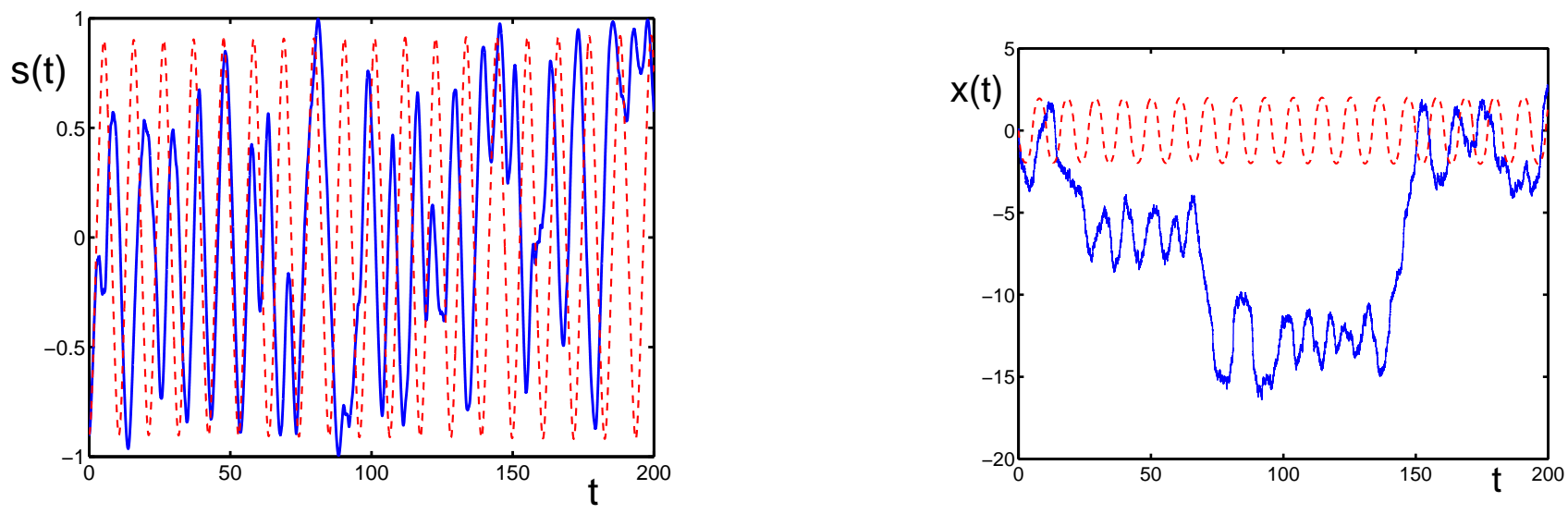

Figure 3: Supercritical regime with $b=0.5>b_{c}=0.282$. Population imbalance $s(t)$ and phase difference $x(t)$, as functions of dimensionless time, for $\sigma=0.5$ (solid line) and $\sigma=0$ (dashed line). 

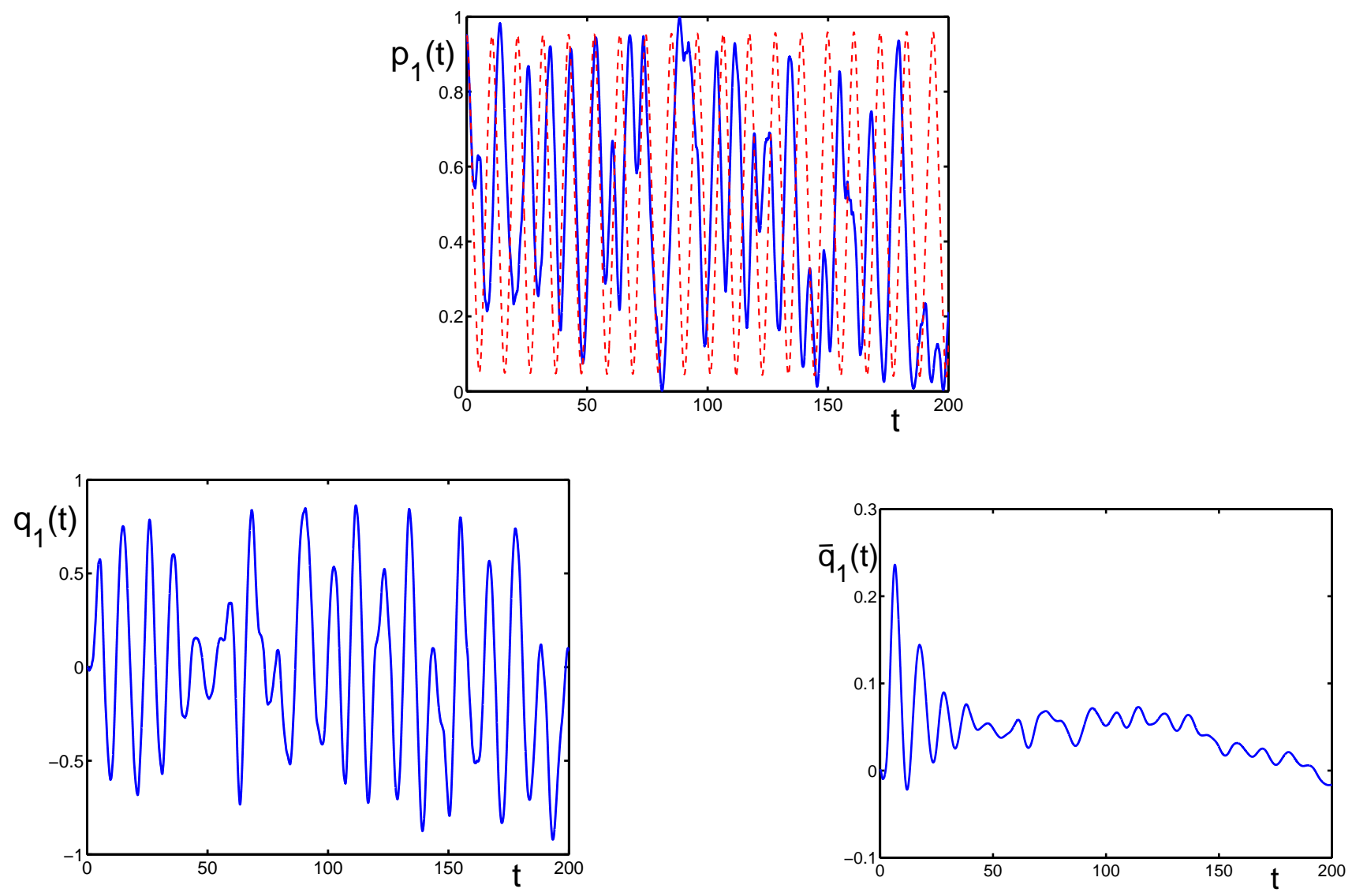

Figure 4: Supercritical regime with $b=0.5>b_{c}=0.282$. Ground-state probability $p_{1}(t)$ for $\sigma=0.5$ (solid line) and $\sigma=0$ (dashed line); interference factor $q_{1}(t)$ and mean interference factor $\bar{q}_{1}(t)$ for $\sigma=0.5$. 